\title{
SOME SURVEY REMARKS ON WHITHAM THEORY AND EM DUALITY
}

\author{
ROBERT CARROLL \\ Mathematics Department, University of Illinois, Urbana, IL 61801 \\ email: rcarroll@symcom.math.uiuc.edu
}

Key words and phrases: Whitham equations, KP and Toda hierarchies, integrability, Riemann surfaces, moduli, $N=2$ supersymmetric gauge theory, Seiberg-Witten differential, monodromy.

\section{INTRODUCTION}

In the last few years there has been enormous progress in string theory, connected with various ideas of duality. Similarly in $N=2$ supersymmetric (susy) gauge theory the idea of electromagnetic (EM) duality emerged in 1994 with the work of Seiberg-Witten [30]. The mathematical consequences of such work have been no less profound than the physical import but a survey is not contemplated here. One theme which has emerged however involves the interaction of ideas of integrability with matrix models, topological field theory (TFT), EM duality, Landau-Ginsburg (LG) models, etc. This is perhaps not surprising in that integrability involving invariant tori etc. seems fundamental in quantization itself but the interaction here is most explicit and inevitable. Without going into a discussion of $N=2$ and $N=1$ susy let us simply say that the low energy effective action for an $N=2$ susy Yang-Mills (YM) theory with gauge group $S U(2)$ for example is described by a holomorphic prepotential $\mathcal{F}$ (see [4, 9, 30] for a discussion and [19] for susy - we will not give extensive references to the physics here). This theory has a scalar potential $V(\phi)=0$ in the vacuum and there are generally nonvanishing $\phi$ for which this occurs. Setting $\phi=(1 / 2) a \sigma_{3}\left(\sigma_{3}=\left(\begin{array}{cc}1 & 0 \\ 0 & -1\end{array}\right)\right)$ and $u=<\operatorname{Tr} \phi^{2}>=(1 / 2) a^{2}(<>\sim$ vacuum expectation value or vev, where $\sim$ is used for "corresponds to" or sometimes "is asymptotic to"), the complex parameter $u$ labels inequivalent vacua and the manifold of gauge inequivalent vacua is called the moduli space $\mathcal{M}$ of the theory (here $u$ is a coordinate on $\mathcal{M} \sim \mathbf{C}$ with punctures or singularities). A metric on $\mathcal{M}$ is given locally by $d s^{2}=\Im \mathcal{F}^{\prime \prime}(a) d a d \bar{a}=$ $\Im \tau(a) d a d \bar{a}$ where $\tau(a) \sim(\theta / 2 \pi)+\left(4 \pi i / g^{2}\right)$ is the complex coupling constant. One writes $a^{D}=$ $\left(\partial \mathcal{F} / \partial a\right.$ (with $\left.\left.<\phi^{D}\right\rangle=(1 / 2) a^{D} \sigma_{3}\right)$ and the superscript $D$ indicates duality here. There is a Legendre transformation connecting dual superfields, etc. and $d s^{2}$ becomes $d s^{2}=\Im d a^{D} d \bar{a}$. On $\mathcal{M}$ physical arguments show that $a(u), a^{D}(u)$ are single valued except for monodromies around say $\left(u_{0},-u_{0}, \infty\right)$ (e.g. $\left(a^{D}, a\right)^{T} \rightarrow M_{\infty}\left(a^{D}, a\right)^{T}$ for $\left.M_{\infty}=\left(\begin{array}{cc}-1 & 2 \\ 0 & -1\end{array}\right)\right)$ and one picks $u_{0}=1$ by renormalization arguments. This leads to a realization of the monodromies by a differential equation ( $)\left(-D_{z}^{2}+V(z)\right) \vec{\psi}(z)=0, \vec{\psi}=\left((i / 2) a,-i a^{D}\right)^{T}$ and this can be rewritten as a hypergeometric equation from which one obtains

$$
\begin{gathered}
a_{D}(u)=i \psi_{2}(u)=i \frac{u-1}{2} F\left(\frac{1}{2}, \frac{1}{2}, 2, \frac{1-u}{2}\right)=\frac{\sqrt{2}}{\pi} \int_{1}^{u} \frac{d x \sqrt{x-u}}{\sqrt{x^{2}-1}} \\
a(u)=-2 i \psi_{1}(u)=\sqrt{2}(u+1)^{\frac{1}{2}} F\left(-\frac{1}{2}, \frac{1}{2}, 1, \frac{2}{u+1}\right)=\frac{\sqrt{2}}{\pi} \int_{-1}^{1} \frac{d x \sqrt{x-u}}{\sqrt{x^{2}-1}}
\end{gathered}
$$


One can eliminate $u$ to get e.g. $a^{D}(a)$. The integral formulas in (1.1) are easily recognized as arising from an elliptic curve (take branch cuts $(-1,1)$ and $(u, \infty)$ in $\mathbf{C}$ and construct a two sheeted Riemann surface of genus one (torus)). Take homology cycles $A \sim$ cycle $1 \rightarrow u$ on one sheet and $u \rightarrow 1$ on the other, with $B \sim$ cycle around $(-1,1)$ and define now

$$
a=\oint_{A} d \lambda ; a^{D}=\oint_{B} d \lambda ; d \lambda=\frac{1}{\pi \sqrt{2}} \frac{\sqrt{z-u}}{\sqrt{z^{2}-1}} d z
$$

This differential $d \lambda=d \lambda_{S W}$ is the Seiberg-Witten (SW) differential and the integrals in (1.2) are period integrals which satisfy $(\mathbf{\$})$ under the label of Picard-Fuchs (PF) equations. The correct monodromies are obtained directly (cf. also [32] for an interesting use of monodromy ideas). We will indicate how all this is related to integrable systems and the Whitham equations.

\section{WHITHAM EQUATIONS}

We will work in the context of algebro-geometric solutions of integrable partial differential equations (PDE) which involves the following ingredients (cf. [3, 8, 14]). Take an arbitrary Riemann surface $\Sigma$ of genus $g$, pick a point $Q$ and a local variable $1 / k$ near $Q$ such that $k(Q)=\infty$, and, for illustration, take $q(k)=k x+k^{2} y+k^{3} t$. Let $D=P_{1}+\cdots+P_{g}$ be a non-special divisor of degree $g$ and write $\psi$ for the (unique up to a constant multiplier by virtue of the Riemann-Roch theorem) Baker-Akhiezer (BA) function characterized by the properties that $\psi$ is meromorphic on $\Sigma$ except for $Q$ where $\psi(P) \exp (-q(k))$ is analytic and $(*) \psi \sim \exp (q(k))\left[1+\sum_{1}^{\infty}\left(\xi_{j} / k^{j}\right)\right]$ near $Q ;$ on $\Sigma / Q, \psi$ has poles at the $P_{i}$. In fact $\psi$ can be taken in the form $\left(P \in \Sigma, P_{0} \neq Q\right)$

$$
\psi(x, y, t, P)=\exp \left[\int_{P_{0}}^{P}\left(x d \Omega^{1}+y d \Omega^{2}+t d \Omega^{3}\right)\right] \cdot \frac{\Theta\left(\mathcal{A}(P)+x U+y V+t W+z_{0}\right)}{\Theta\left(\mathcal{A}(P)+z_{0}\right)}
$$

where $d \Omega^{1}=d k+\cdots, d \Omega^{2}=d\left(k^{2}\right)+\cdots, d \Omega^{3}=d\left(k^{3}\right)+\cdots, U_{j}=\int_{B_{j}} d \Omega^{1}, V_{j}=\int_{B_{j}} d \Omega^{2}, W_{j}=$ $\int_{B_{j}} d \Omega^{3}(j=1, \cdots, g), z_{0}=-\mathcal{A}(D)-K$, and $\Theta$ is the Riemann theta function. Here the $d \Omega_{j}$ are meromorphic differentials of second kind normalized via $\int_{A_{k}} d \Omega_{j}=0\left(A_{j}, B_{j}\right.$ are canonical homology cycles) and we note that $x d \Omega^{1}+y d \Omega^{2}+t d \Omega^{3} \sim d q(k)$ normalized; $\mathcal{A}$ is the Abel-Jacobi map $(\mathcal{A}(P)=$ $\int^{P} d \omega_{k}$ where the $d \omega_{k}$ are normalized holomorphic differentials, $\left.k=1, \cdots, g, \int_{A_{j}} d \omega_{k}=\delta_{j k}\right)$, and $K=\left(K_{j}\right) \sim$ Riemann constants $\left(2 K=-\mathcal{A}\left(K_{\Sigma}\right)\right.$ where $K_{\sigma}$ is the canonical class of $\Sigma \sim$ equivalence class of meromorphic differentials) so $\Theta\left(\mathcal{A}(P)+z_{0}\right.$ ) has exactly $g$ zeros (or vanishes identically). The paths of integration are to be the same in computing $\int_{P_{0}}^{P} d \Omega^{i}$ or $\mathcal{A}(P)$ and it is shown in [3, 0, 14] that $\psi$ is well defined (i.e. path independent). Then the $\xi_{j}$ in $\left(^{*}\right)$ can be computed formally and one determines Lax operators $L$ and $A$ such that $\partial_{y} \psi=L \psi$ with $\partial_{t} \psi=A \psi$. Indeed, given the $\xi_{j}$ write $u=-2 \partial_{x} \xi_{1}$ with $w=3 \xi_{1} \partial_{x} \xi_{1}-3 \partial_{x}^{2} \xi_{1}-3 \partial_{x} \xi_{2}$. Then formally, near $Q$, one has $\left(-\partial_{y}+\partial_{x}^{2}+u\right) \psi=O(1 / k) \exp (q)$ and $\left(-\partial_{t}+\partial_{x}^{3}+(3 / 2) u \partial_{x}+w\right) \psi=O(1 / k) \exp (q)$ (i.e. this choice of $u, w$ makes the coefficients of $k^{n} \exp (q)$ vanish for $\left.n=0,1,2,3\right)$. Now define $L=\partial_{x}^{2}+u$ and $A=\partial_{x}^{3}+(3 / 2) u \partial_{x}+w$ so $\partial_{y} \psi=L \psi$ and $\partial_{t} \psi=A \psi$. This follows from the uniqueness of BA functions with the same essential singularity and pole divisors (Riemann-Roch). Then we have, via compatibility $L_{t}-A_{y}=[A, L]$, a KP equation $(3 / 4) u_{y y}=\partial_{x}\left[u_{t}-(1 / 4)\left(6 u u_{x}+u_{x x x}\right)\right]$ and therefore such KP equations arise automatically from a Riemann surface and are parametrized by nonspecial divisors or equivalently by points in general position on the Jacobian variety $J(\Sigma)$. The flow variables $x, y, t$ arise via $q(k)$ and then miraculously reappear in the theta function via $x U+y V+t W$; thus the Riemann surface itself contributes to establish these as linear flow variables on the Jacobian and in a certain sense defines the flow variables. The pole positions $P_{i}$ do not vary with $x, y, t$ and (†) $u=2 \partial_{x}^{2} \log \Theta\left(x U+y V+t W+z_{0}\right)+c$ exhibits $\Theta$ as a tau function. 
We recall also that a divisor $D^{*}$ of degree $g$ is dual to $D$ (relative to $Q$ ) if $D+D^{*}$ is the null divisor of a meromorphic differential $d \hat{\Omega}=d k+\left(\beta / k^{2}\right) d k+\cdots$ with a double pole at $Q$ (look at $\zeta=1 / k$ to recognize the double pole). Thus $D+D^{*}-2 Q \sim K_{\Sigma}$ so $\mathcal{A}\left(D^{*}\right)-\mathcal{A}(Q)+K=-[\mathcal{A}(D)-\mathcal{A}(Q)+K]$. One can define then a function $\left.\psi^{*}(x, y, t, P)=\exp \left(-k x-k^{2} y-k^{3} t\right)\left[1+\xi_{1}^{*} / k\right)+\cdots\right]$ based on $D^{*}$ (dual BA function) and a differential $d \hat{\Omega}$ with zero divisor $D+D^{*}$, such that $\phi=\psi \psi^{*} d \hat{\Omega}$ is meromorphic, having for poles only a double pole at $Q$ (the zeros of $d \hat{\Omega}$ cancel the poles of $\psi \psi^{*}$ ). Thus $\psi \psi^{*} d \hat{\Omega} \sim \psi \psi^{*}\left(1+\left(\beta / k^{2}+\cdots\right) d k\right.$ is meromorphic with a second order pole at $\infty$, and no other poles. For $L^{*}=L$ and $A^{*}=-A+2 w-(3 / 2) u_{x}$ one has then $\left(\partial_{y}+L^{*}\right) \psi^{*}=0$ and $\left(\partial_{t}+A^{*}\right) \psi^{*}=0$. Now the prescription above seems to specify for $\psi^{*}\left(\vec{U}=x U+y V+t W, z_{0}^{*}=-\mathcal{A}\left(D^{*}\right)-K\right)$

$$
\psi^{*} \sim e^{-\int_{P_{o}}^{P}\left(x d \Omega^{1}+y d \Omega^{2}+t d \Omega^{3}\right)} \cdot \frac{\Theta\left(\mathcal{A}(P)-\vec{U}+z_{0}^{*}\right)}{\Theta\left(\mathcal{A}(P)+z_{0}^{*}\right)}
$$

In any event the message here is that for any Riemann surface $\Sigma$ one can produce a BA function $\psi$ with assigned flow variables $x, y, t, \cdots$ and this $\psi$ gives rise to a (nonlinear) KP equation with solution $u$ linearized on the Jacobian $J(\Sigma)$.

One knows that hyperelliptic curves play a special role in the theory of algebraic curves and Riemann surfaces. For hyperelliptic Riemann surfaces one can pick any $2 g+2$ points $\lambda_{j} \in \mathbf{P}^{1}$ and there will be a unique hyperelliptic curve $\Sigma_{g}$ with a 2-fold map $f: \Sigma_{g} \rightarrow \mathbf{P}^{1}$ having branch locus $B=\left\{\lambda_{j}\right\}$. Since any 3 points $\lambda_{i}, \lambda_{j}, \lambda_{k}$ can be sent to $0,1, \infty$ by an automorphism of $\mathbf{P}^{1}$ the general hyperelliptic surface of genus $g$ can be described by $(2 g+2)-3=2 g-1$ points on $\mathbf{P}^{1}$. Since $f$ is unique up to an automorphism of $\mathbf{P}^{1}$ any hyperelliptic $\Sigma_{g}$ corresponds to only finitely many such collections of $2 g-1$ points so locally there are $2 g-1$ (moduli) parameters. Since the moduli space of algebraic curves has dimension $3 g-3$ one sees that for $g \geq 3$ the generic Riemann surface is nonhyperelliptic whereas for $g=2$ all Riemann surfaces are hyperelliptic (with 3 moduli). For $g=1$ we have tori or elliptic curves with one modulus $\tau$ and $g=0$ corresponds to $\mathbf{P}^{1}$. In many papers on soliton mathematics and integrable systems one takes real distinct branch points $\lambda_{j}, 1 \leq j \leq 2 g+1$, and $\infty$, with $\lambda_{1}<\lambda_{2}<\cdots<\lambda_{2 g+1}<\infty$ and $\mu^{2}=\prod_{1}^{2 g+1}\left(\lambda-\lambda_{j}\right)=P_{2 g+1}\left(\lambda, \lambda_{j}\right)$ as the defining equation for $\Sigma_{g}$. Evidently one could choose $\lambda_{1}=0, \lambda_{2}=1$ in addition so for $g=1$ we could use $0<1<u<\infty$ for a familiar parametrization with elliptic integrals, etc. One can take $d \lambda / \mu, \lambda d \lambda / \mu, \cdots, \lambda^{g-1} d \lambda / \mu$ as a basis of holomorphic differentials on $\Sigma_{g}$ but usually one takes linear combinations of these denoted by $d \omega_{j}, 1 \leq j \leq g$, normalized via $\oint_{A_{i}} d \omega_{j}=\delta_{i j}$, with period matrix defined via $\oint_{B_{i}} d \omega_{j}=b_{i j}$. The matrix $\Pi=\left(\Pi_{i j}\right)$ is symmetric with $\Im \Pi>0$ and it determines the curve. One will also want to consider another representation of hyperelliptic curves of genus $g$ via $\mu^{2}=\prod_{0}^{2 g+1}\left(\lambda-\lambda_{j}\right)=P_{2 g+2}\left(\lambda, \lambda_{j}\right)$ where $\infty$ is now not a branch point and there are two points $\mu_{ \pm}$ corresponding to $\lambda=\infty$.

We extract now from [0] which provides some embellishments to [20, 25]. Variations of the Whitham-Bogoliubov averaging procedure have proved to be of considerable interest and a very nice introduction to averaging for $\mathrm{KdV}$ appears in [18]. Thus one considers modulated waves based on a potential $u\left(\theta_{i}, \lambda_{j}\right)$ where the fast variables $x, t$ occur via $\theta_{i}=x U_{i}+t W_{i}$ and the slow variables occur in the moduli (here branch points) $\lambda_{j}=\lambda_{j}(X, T)$ (for $\mathrm{KdV}$ one is dealing with hyperelliptic surfaces). The averaging procedure looks for example at $<f(u)>\sim \lim _{L \rightarrow \infty}(1 / 2 L) \int_{-L}^{L} f(u)\{d x$ or $d t\}$ for suitable functions $f(u)$, and, assuming incommensurable frequencies, ergodicity implies $\langle f(u)\rangle=$ $(1 / 2 \pi)^{N} \int_{0}^{2 \pi} \cdots \int_{0}^{2 \pi} f\left(u\left(\theta_{i}, \lambda_{j}\right) \prod_{1}^{2 N} d \theta_{i}\right.$, where the slow variables are fixed. This is made more explicit below. One should perhaps contrast this procedure explicitly with dispersionless limit techniques which arise in the genus zero situation. For $\mathrm{KdV} u_{t}+(3 / 2) u u_{x}-(1 / 4) u_{x x x}=0$ (based e.g. on $\left.L^{2}=\partial^{2}-u, B=\partial^{3}-(3 / 2) u \partial-(3 / 4) u_{x}, \partial_{t} L^{2}=\left[B, L^{2}\right]\right)$ one writes $\epsilon t=T, \epsilon x=X$ to obtain 
formally $u_{T}+(3 / 2) u u_{X}=0$ as $\epsilon \rightarrow 0$ (dispersionless $\mathrm{KdV}$ or Euler equation). The background $\mathrm{dKdV}$ theory for Lax operators involves $L \psi=\lambda \psi$ with $\psi=\exp [(1 / \epsilon) S(X, T)]$ so for $P=\partial_{X} S, \lambda=$ $P^{2}-U(X, T)$ where one usually assumes $u(x, t)=U(X, T)+O(\epsilon)$. This last step is never really discussed adequately in work on (algebraic type) dispersionless theory and we make a brief comment here. In question is the behavior of $u(x, t)=u(X / \epsilon, T / \epsilon)$ as $x, t \rightarrow \infty$ (or $\epsilon \rightarrow 0$ with $X, T$ fixed) and there is a priori no modulated wave or quasiperiodic situation to make the analysis easier. A detailed analysis of $\mathrm{KdV} \rightarrow$ Euler for example is developed in classical work of Lax, Levermore, and Venakides for example (see [28] for references and discussion) and a weak limiting procedure is justified in many situations. Similar analysis also applies to nonlinear Schrödinger and Toda equations. There is however no general theory here and the analysis becomes deep and technical. The moral is that the algebraic limiting procedure is justified sometimes in realistic situations and we simply assume it to be OK whenever it arises. The averaging procedure for quasiperiodic modulated waves on the other hand seems to be quite generally applicable.

Let us sketch now the averaging procedure following [25] with clarifications as in [7, 20] (cf. also [26]). Consider KP in the form $3 u_{y} y+\partial_{x}\left(4 u_{t}-6 u u_{x}+u_{x x x}\right)=0$ via compatibility $\left[\partial_{y}-L, \partial_{t}-A\right]=0$ where $L=\partial^{2}-u$ and $A=\partial^{3}-(3 / 2) u \partial+w\left(u \rightarrow-u\right.$ in (2.1). We have then $\left(\partial_{y}-L\right) \psi=0$ with $\left(\partial_{t}-A\right) \psi=0$ and for the adjoint or dual wave function $\psi^{*}$ one writes $\psi^{*} L=-\partial_{y} \psi^{*}$ with $\psi^{*} A=-\partial_{t} \psi^{*}$ where $\psi^{*}\left(f \partial^{j}\right) \equiv(-\partial)^{j}\left(\psi^{*} f\right)$. We can use formulas (cf. (2.1) and (2.2)) $\psi=$ $e^{p x+E y+\Omega t} \cdot \phi(U x+V y+W t, P)$ and $\psi^{*}=e^{-p x-E y-\Omega t} \cdot \phi^{*}(-U x-V y-W t, P)$ to isolate the quantities of interest in averaging (here $p=p(P), E=E(P), \Omega=\Omega(P)$, etc.) We think here of a general Riemann surface $\Sigma_{g}$ with holomorphic differentials $d \omega_{k}$ and quasi-momenta and quasi-energies of the form $d p=d \Omega^{1}, d E=d \Omega^{2}, d \Omega=d \Omega^{3}, \cdots\left(p=\int_{P_{0}}^{P} d \Omega^{1}\right.$ etc. $)$ where the $d \Omega^{j}=d \Omega_{j}=d\left(\lambda^{j}+O\left(\lambda^{-1}\right)\right)$ are meromorphic differentials of the second kind. Following [25] we normalize now via $\Re \int_{A_{j}} d \Omega^{k}=$ $\Re \int_{B_{j}} d \Omega^{k}=0$. Then write e.g. $U_{k}=(1 / 2 \pi i) \oint_{A_{k}} d p$ and $U_{k+g}=-(1 / 2 \pi i) \oint_{B_{k}} d p(k=1, \cdots, g)$ with similar stipulations for $V_{k} \sim \oint d \Omega^{2}, W_{k} \sim \oint d \Omega^{3}$, etc. This leads to real $2 g$ period vectors and evidently one could also normalize via $\oint_{A_{m}} d \Omega^{k}=0$ or $\Im \oint_{A_{m}} d \Omega^{k}=\Im \oint_{B_{m}} d \Omega^{k}=0$. It is then immediate that $\left(\psi^{*} L\right) \psi=\psi^{*} L \psi+\partial_{x}\left(\psi^{*}\left(L^{1} \psi\right)\right)+\partial_{x}^{2}\left(\psi^{*}\left(L^{2} \psi\right)\right)+\cdots$ where e.g. $\psi^{*}\left(u \partial^{j}\right)=(-\partial)^{j}\left(\psi^{*} u\right)$. More precisely, for $A=\sum_{0}^{k} a_{i} \partial^{i}$, one has $\psi^{*} A=\sum_{0}^{k}(-\partial)^{i}\left(\psi^{*} a_{i}\right)$ and $\left(\psi^{*} A\right) \psi=\sum_{0}^{k} \partial^{j}\left(\psi^{*}\left(A^{j} \psi\right)\right)$ with $A^{0}=A, A^{1}=-\sum_{1}^{k} i a_{i} \partial^{i-1}, A^{2}=\sum_{2}^{k}[i(i-1) / 2] a_{i} \partial^{i-2}, \ldots$. For $L$ and $A$ as in KP above $L^{1}=-2 \partial, L^{2}=1$ and $A^{1}=-3 \partial^{2}+(3 / 2) u, A^{2}=3 \partial, A^{3}=-1$.

Now for averaging we think of $u=u_{0}([1 / \epsilon] S(X, Y, T) \mid I(X, Y, T))+\epsilon u_{1}(x, y, t)+\epsilon^{2} u_{2}(x, y, t)+\cdots$ ( $I \sim$ moduli and $S \sim$ action in some sense) with $\partial_{X} S=U, \partial_{Y} S=V$, and $\partial_{T} S=W$. We think of expanding about $u_{0}$ with $\partial_{x} \rightarrow \partial_{x}+\epsilon \partial_{X}$. This step will cover both $x$ and $X$ dependence for subsequent averaging. Then look at the compatibility condition $(\ddagger): \partial_{t} L-\partial_{y} A+[L, A]=0$. We will want the term of first order in $\epsilon$ upon writing e.g. $L=L_{0}+\epsilon L_{1}+\cdots$ and $A=A_{0}+\epsilon A_{1}+\cdots$ where $L_{0}, A_{0}$ are to depend on the slow variables $X, Y, T$, and this gives $\partial_{t} L_{1}-\partial_{y} A_{1}+\left[L_{0}, A_{1}\right]+\left[L_{1}, A_{0}\right]+F=$ $0 ; F=\partial_{T} L-\partial_{Y} A-L^{1} \partial_{X} A+A^{1} \partial_{X} L$. Thus $F$ is the first order term involving derivatives in the slow variables. Next one writes $\partial_{t}\left(\psi^{*}\left(L_{1} \psi\right)\right)-\partial_{y}\left(\psi^{*}\left(A_{1} \psi\right)\right)=\psi^{*}\left\{L_{1 t}-A_{1 y}+\left[L_{1}, A\right]+\left[L, A_{1}\right]\right\} \psi=$ $\psi^{*}\left(\partial_{t} L_{1}-\partial_{y} A_{1}+\left[L_{0}, A_{1}\right]+\left[L_{1}, A_{0}\right]\right) \psi+\partial_{x}(\cdots)$ and via ergodicity in $x, y$, or $t$ flows, averaging of derivatives in $x, y$, or $t$ gives zero so one obtains the Whitham equations in the form $\left\langle\psi^{*} F \psi\right\rangle=0$ (this represents the first order term in $\epsilon$ - the slow variables are present in $L_{0}, A_{0}, \psi$, and $\psi^{*}$ ). In order to spell this out in [20] one imagines $X, Y$, or $T$ as a parameter $\xi$ and considers $L(\xi), A(\xi)$, etc. (in their perturbed form) with $\psi(\xi)=e^{p(\xi) x+E(\xi) y+\Omega(\xi) t} \cdot \phi(U(\xi) x+V(\xi) y+W(\xi) t \mid I(\xi)$ ) and $\psi^{*}=\exp (-p x-E y-\Omega t) \phi^{*}(-U x-V y-W t \mid I)$ (no $\xi$ variation - i.e. assume $p, E, \Omega, U, V, W, I$ fixed). Also note that $x, y, t$ and $X, Y, T$ can be considered as independent variables. Upon differentiating 
various expressions with respect to $x, y, t$ and $\xi$ and combining one obtains then

$$
d \Omega<\psi^{*} \psi>=-d p<\psi^{*} A^{1} \psi>; d E<\psi^{*} \psi>=-d p<\psi^{*} L^{1} \psi>
$$

and a version of the Whitham equations in the form

$$
p_{T}=\Omega_{X} ; p_{Y}=E_{X} ; E_{T}=\Omega_{Y}
$$

Note also from $\partial_{X} S=U, \partial_{Y} S=V$, and $\partial_{T} S=W$ one has compatibility relations $\partial_{Y} U=$ $\partial_{X} V, \partial_{T} U=\partial_{X} W$, and $\partial_{T} V=\partial_{Y} W$. We mention also an equation obtained along the way which will be important in the analysis of [26], namely

$$
\begin{gathered}
<\psi^{*}\left(L^{1} \partial_{X} A-A^{1} \partial_{X} L\right) \psi>=\partial_{X} \Omega<\psi^{*} L^{1} \psi>-\partial_{X} E<\psi^{*} A^{1} \psi>+ \\
+\partial_{X} W \cdot<\psi^{*} L^{1} \psi_{\theta}>-\partial_{X} V \cdot<\psi^{*} A^{1} \psi_{\theta}>
\end{gathered}
$$

We feel that this derivation is important since it exhibits again (as in [18] for the KdV situation) the role of "square eigenfunctions" (now in the form $\psi^{*} \psi$ ) in dealing with averaging processes (cf. [0], 10] for more on $\psi^{*} \psi$ and [7] for details on averaging).

Next, d'après []], we give some formulas for differentials followed by a few remarks on hyperelliptic situations. The KP flows can be written as $\partial_{n} u=K_{n}(u)$ where the $K_{n}$ are symmetries satisfying (in the notation of [10]) the linearized KP equation $\partial_{3} \beta=(1 / 4) \partial^{3} \beta+3 \partial(u \beta)+(3 / 4) \partial^{-1} \partial_{2}^{2} \beta=K^{\prime}[\beta]$. The conserved densities or gradients $\gamma$ satisfy the adjoint linearized KP equation $\partial_{3} \gamma=(1 / 4) \partial^{3} \gamma+$ $3 u \partial \gamma+(3 / 4) \partial^{-1} \partial_{2}^{2} \gamma$. Then, replacing the square eigenfunctions of $\mathrm{KdV}$ theory by $\psi \psi^{*}$ one has e.g. $\psi \psi^{*}=\sum_{0}^{\infty} s_{n} \lambda^{-n}$ where $s_{n} \sim \gamma_{n}$. Further $\partial_{n} u=K_{n+1}=\partial s_{n+1}=\partial \operatorname{Res} L^{n}=\partial \nabla \hat{I}_{n+1}$ where $\nabla f \sim \delta f / \delta u$ (Res $L^{n}=n H_{n-1}^{1}$ is generally used in the multipotential theory). We are thinking here in a single potential theory where all potentials $u_{i}$ in $L=\partial+\sum_{1}^{\infty} u_{i+1} \partial^{-i}$ are expressed in terms of $u_{2}=u$ via operations with $\partial$ and $\partial^{-1}$. One uses here the Poisson bracket $\{f, g\}=$ $\iint(\delta f / \delta u) \partial(\delta g / \delta u) d x d y$ (Gardner bracket). Thus one has $s_{n+1} \sim \gamma_{n+1} \sim \nabla \hat{I}_{n+1}$ as conserved gradients satisfying the adjoint linear KP equation $\left.{ }^{* *}\right) \partial_{t} \gamma=(1 / 4) \partial^{3} \gamma+3 u \partial \gamma+(3 / 4) \partial^{-1} \partial_{y}^{2} \gamma$. Following [18] one linearizes around a fixed finite zone solution $u$ in the adjoint linear KP equation and puts this $u$ into the $\hat{I}_{j}$ etc.; then averaging over the $\theta_{i}$ variables is carried out as before. Also from $\psi \psi^{*}=\sum_{1}^{\infty}\left(s_{n} / \lambda^{n}\right)$ (cf. [10]) we can write $<\psi \psi^{*}>=\sum_{1}^{\infty}\left(<s_{n}>/ \lambda^{n}\right)$ Such a series is natural from asymptotic expansions but when $\psi, \psi^{*}$ are written in terms of theta functions it requires expansion of the theta functions in $1 / \lambda$ (such expansions are documented in [14], p. 49 for example). It is now natural to ask whether one can express $d p, d E, d \Omega$ from (2.3) in more detail. Some order of magnitude considerations suggest $d p \sim<\psi \psi^{*}>d \lambda$ near $\infty$. To prove this we refer to [10] for background and notation (cf. also [11, 12] for dispersionless genus zero situations). We write $\partial=L+\sum_{1}^{\infty} \sigma_{j}^{1} L^{-j}$ which implies that $(\partial \psi / \psi)=\lambda+\sum_{1}^{\infty} \sigma_{j}^{1} \lambda^{-j}$. Then $(\log \psi)_{x}=\lambda+\sum_{1}^{\infty} \sigma_{j}^{1} \lambda^{-j}$ and $\overline{(\log \psi)_{x}} \sim<(\log \psi)_{x}>=p=\lambda+\sum_{1}^{\infty}<\sigma_{j}^{1}>\lambda^{-j}$. But $s_{n+1}=-n \sigma_{n}^{1}-\sum_{1}^{n-1} \partial_{j} \sigma_{n-j}^{1}$ and one obtains $<s_{n+1}>=-n<\sigma_{n}^{1}>-\sum_{1}^{n-1}<\partial_{j} \sigma_{n-j}^{1}>=-n<\sigma_{n}^{1}>$. Thus $d p=d \lambda-\sum_{1}^{\infty} j<\sigma_{j}^{1}>\lambda^{-j-1} d \lambda=$ $d \lambda+\sum_{1}^{\infty}<s_{j+1}>\lambda^{-j-1} d \lambda=<\psi \psi^{*}>$ since $\psi \psi^{*}=\sum_{0}^{\infty} s_{n} \lambda^{-n}$. The normalization is built into this construction. It is clear that $d p=<\psi \psi^{*}>d \lambda$ cannot hold globally since $\left\langle\psi \psi^{*}>\right.$ should have poles at $D+D^{*}$ and the correct global statement follows from [25, 26], namely $\left(d p /<\psi \psi^{*}>\right)=d \hat{\Omega}$ where $d \hat{\Omega}$ is the unique meromorphic differential with a double pole at $\infty$ and zeros at $D+D^{*}$. To see this note that $d p /\left\langle\psi \psi^{*}>\right.$ will have zeros at the poles of $\psi \psi^{*}$ (i.e. at $D+D^{*}$ ) and a double pole at $\infty$; this characterizes $d \hat{\Omega}$.

In summary we see that the quantity $\psi^{*} \psi$ is seen to determine the Whitham hierarchy (2.4) and 
the differentials $d p, d E, d \Omega$, etc. via

$$
\frac{d p}{<\psi \psi^{*}>}=-\frac{d E}{<\psi^{*} L^{1} \psi>}=-\frac{d \Omega}{<\psi^{*} A^{1} \psi>}=d \hat{\Omega}
$$

In the hyperelliptic case with say $R(\Lambda)=\prod_{1}^{2 g+1}\left(\Lambda-\Lambda_{j}\right)$ one can explicitly write down the differentials $d p, d \Omega$, etc. as $d p=P(\Lambda) / \sqrt{R}, P(\Lambda)=\Lambda^{g}+\sum_{1}^{g} a_{i} \Lambda^{g-i}$ and $d \Omega=Q(\Lambda) / \sqrt{R}, Q(\Lambda)=$ $6 \Lambda^{g+1}+\sum_{0}^{g} b_{j} \Lambda^{g-j}$. Now from (2.4) (\$) $\partial_{T} d p=\partial_{X} d \Omega=(d \Omega / d p) \partial_{X} d p$. The moduli here are the branch points $\Lambda_{j}$ which depend on the slow variables so writing out (\$), multiplying by $\left(\Lambda-\Lambda_{i}\right)^{3 / 2}$, and passing to limits $\Lambda \rightarrow \Lambda_{i}$ yields the Whitham equations as equations for Riemann invariants $\Lambda_{k}$ in the form $\partial_{T} \Lambda_{k}=v_{k}\left(\Lambda_{1}, \cdots, \Lambda_{2 g+1}\right) \partial_{X} \Lambda_{k}$ where the characteristic velocities $v_{k}=\left.(d \Omega / d p)\right|_{\Lambda=\Lambda_{k}}$ have an elementary expression (cf. [0, 18]). One can also use the integrals of motion $\left(\sim \hat{I}_{n}\right.$ above $)$ as moduli (see remarks later).

\section{ACTION AND PREPOTENTIAL}

The viewpoint of [23] for example is now to look at SW theory in terms of a renormalization group map $\left\{G, \tau, m, h_{i}\right\} \rightarrow\left\{a_{i}(h), \mathcal{F}\left(a_{i}\right)\right\}$ where $G \sim$ gauge group, $\tau \sim$ bare coupling constant, $m \sim$ mass scale, $h_{i} \sim$ symmetry breaking vev's, $a_{i}(h) \sim$ background "fields", and $\mathcal{F} \sim$ prepotential (with $a_{i}=\partial \mathcal{F} / \partial a_{i}$, etc.). This map is then decomposed as $\{G, \tau, m, h\} \rightarrow\left\{\Sigma, d \mathcal{S}_{\min }\right\} \rightarrow\left\{a_{i}(h), \mathcal{F}\left(a_{i}\right)\right\}$ where $\Sigma$ is a Riemann surface and $d \mathcal{S}_{\text {min }}$ is a meromorphic one form on $\Sigma$ such that $\partial d \mathcal{S} / \partial h_{i}$ is holomorphic. Since the genus of $\Sigma$ may be larger than $r_{G}=\operatorname{rank}(G)$ one must make some adjustments for which we refer to [23]. Roughly $d \mathcal{S}_{\min } \sim d \lambda_{S W}$ (modulo a few nonzero times - cf. [?, 22, 23]) and $h \sim$ moduli as will be indicated. The first map to $\left\{\Sigma, d \mathcal{S}_{\min }\right\}$ involves quite general ideas and can be viewed via a spectral cover of some base space $E(\tau)$ and an integrable system determined by a (matrix) Lax operator based on $E(\tau)$ (generally $E(\tau) \sim \mathbf{P}^{1}$ or an elliptic curve). Thus one looks at $\Sigma: \operatorname{det}(t-L(z))=0$ which defines $\Sigma$ as a ramified cover of $E$ and the integrals of motion (or Casimirs) for the corresponding integrable system will be identified with moduli $h_{i}$. This is in keeping with the spirit of averaging in Section 2. In this context $d \lambda_{S W} \sim t d \omega$ where $d \omega$ is (the) normalized holomorphic differential on $E(\tau)$ and this will be clarified below (cf. [?, 13, 23, 26]). Regarding the second map the action integrals $a_{i}=S_{A_{i}}=\oint_{A_{i}} d \lambda_{S W}$ and $a_{i}^{D}=S_{B_{i}}=\oint_{B_{i}} d \lambda_{S W}$ for $\left(A_{i}, B_{i}\right)$ a canonical homology basis will play a fundamental role. To assist in the determination of $d \lambda_{S W}$ we recall that the monodromy for period integrals $\oint_{C} d \omega$ ( $d \omega_{i}$ a basis of normalized holomorphic differentials) is often obtained from the physics and one will have PF equations for period integrals, etc. Thus it will be sufficient to have $\partial d \lambda_{S W} / \partial h_{k}=\sum_{1}^{g} \alpha_{k j} d \omega_{j}$ so e.g. $\partial a_{i} / \partial h_{k}=\oint_{A_{i}} \sum \alpha_{k j} d \omega_{j}=\sum \alpha_{k j} \delta_{j i}=\alpha_{k i}$ and $\partial a_{i}^{D} / \partial h_{k}=\oint_{B_{i}} \sum \alpha_{k j} d \omega_{j}=\sum \alpha_{k j} b_{j i}$ where $\left(b_{i j}\right)$ is the period matrix for $\Sigma$ (cf. also [26] and remarks below). For hyperelliptic $\Sigma$ one often takes $\partial d \lambda_{S W} / \partial v_{k}=d \hat{\omega}_{k}=\left(\Lambda^{k} d \Lambda / y\right) \sim$ unnormalized holomorphic differentials $\left(y=\sqrt{R}, R=\prod_{1}^{2 g+2}\left(\Lambda-\Lambda_{i}\right), k=0, \cdots, g-1\right)$ where $v_{k}(1 \leq k \leq g)$ are suitable "flat" moduli (see e.g. [16, 24, 29] for good illustrations and calculations).

Now from [23] (cf. also [9]) one works from a general action $d \mathcal{S}$ and builds in times $T_{n}$ from the beginning. For convenience the development here is in terms of hyperelliptic spectral curves $\Sigma$ which arise automatically from periodic Toda hierarchies (cf. [5, 9, 14]); physically the framework is appropriate for $S U(n) N=2$ susy YM theories with matter for example (and others). Then the $T_{n}$ correspond to quasiclassical (slow) times and the prepotential $\mathcal{F}$ will correspond to $F=\operatorname{logarithm}$ of the tau function for the dispersionless Toda hierarchy. For convenience the development here is in terms of hyperelliptic spectral curves $\Sigma$ which arise automatically from periodic Toda hierarchies (cf. [5, 9, 14]). Thus let $d \omega_{i}$ be canonical holomorphic differentials on $\Sigma_{g}=\Sigma_{g}\left(h_{k}\right)$ where $i=1, \cdots, g$ and $k=1, \cdots, K$. The $h_{k}$ here are to represent the moduli (e.g. they could correspond to branch 
points $\Lambda_{k}$ for hyperelliptic situations). One has then canonical normalizations $\oint_{A_{i}} d \omega_{j}=\delta_{i j}$ and $\oint_{B_{i}} d \omega_{j}=b_{i j}\left(h_{k}\right)$. From the point of view of Yang-Mills (YM) theory the spectral curve $\Sigma_{g}$ is determined by the gauge group $G$ with $h_{k} \sim(1 / k)<\operatorname{Tr} \phi^{k}>$ for a suitable scalar field in the adjoint representation of $G$ which breaks the original gauge symmetry down to $U(1)^{r_{G}}$ (cf. here [4, 13, 30] for details). From the point of view of integrability the $(1 / k)<\operatorname{Tr} \phi^{k}>$ correspond to Hamiltonians (integrals of motion of the integrable system) and one is back to Whitham dynamics. Now in [23] one begins with a meromorphic differential $d S$ on $\Sigma_{g}$ defined (partly) by the requirement $(\bullet)\left(\partial d S / \partial h_{k}\right) \simeq \sum_{1}^{g} \sigma_{k i}^{d S} d \omega_{i} \simeq$ holomorphic differential, where everything depends on the $h_{k}$. Existence of solutions depends on the number $K$ of moduli and one will assume here that $K=g$ (one expects $K>g$ generically). We will anticipate hyperelliptic curves here (e.g. Toda situations) and assume $\Sigma_{g}$ to be given by $\lambda^{2}=\prod_{1}^{2 g+2}\left(\Lambda-\Lambda_{i}\right)=R_{g}(\Lambda)$ for example. Let us pick two points $\lambda_{ \pm} \in \Sigma_{g}$ and use $\lambda$ for a complex coordinate near $\lambda_{+}$or $\lambda_{-}(\lambda$ is correct here, not $\Lambda)$. One defines $d \hat{\Omega}_{n}$ as a solution to $(\bullet)$ satisfying in addition (1) $d \hat{\Omega}_{ \pm n}(n \geq 1)$ has a pole of order $n+1$ at $\lambda_{ \pm}$and no other poles with $d \hat{\Omega}_{ \pm n}(\lambda)= \pm\left[\left(\lambda-\lambda_{ \pm}\right)^{-n-1}+o(1)\right] d \lambda$ and (2) $d \hat{\Omega}_{0}$ has simple poles at both $\lambda_{ \pm}$with residues \pm 1 . Such $d \hat{\Omega}_{n}$ exist for $K=g$ for example and one has then $\left.(\bullet \bullet)\left(\partial d \hat{\Omega}_{n}\right) / \partial h_{k}\right) \simeq \sum_{1}^{g} \sigma_{k i}^{n} d \omega_{i}$. On the other hand differentials $d \Omega_{n}$, satisfying (1) and (2), but not necessarily $(\bullet \bullet)$, arise in Whitham theory with normalization $\oint_{A_{i}} d \Omega_{n}=0$. Note that the conditions (1) and (2) plus normalization define the $d \Omega_{n}$ and then $d \hat{\Omega}_{n} \simeq d \Omega_{n}+\sum_{1}^{g} c_{i}^{n} d \omega_{i}$ must hold with $c_{i}^{n}=\oint_{A_{i}} d \hat{\Omega}_{n}$. One can write then $\kappa_{j}^{n}=\oint_{B_{j}} d \Omega_{n}$ and refer to [29] for the tau function of Toda theory. Then one could use $\psi^{*} \psi$ as in Section 2 for averaging or work directly from an action expression (a nice exposition of Toda averaging is in [5]) and end up with an action term $d \mathcal{S}\left(h_{k}, T_{i}\right)=\sum_{1}^{g} a_{i} d \omega_{i}+\sum_{-\infty}^{\infty} T_{n} d \Omega_{n}$ where the $h_{k}$ are moduli and the $T_{n}$ are slow variables. A priori $a_{i}, d \omega_{i}$, and $d \Omega_{n}$ depend on $h_{k}$ and $T_{n}$ and further analysis is mandatory.

Thus to be more systematic in developing a quasiclassical tau function whose logarithm corresponds to the Seiberg-Witten (SW) prepotential one takes a solution $d \mathcal{S}\left(T_{n} \mid h_{k}\right)$ of $(\bullet)$ (presumed to exist without loss of generality since we have indicated how such creatures could arise from period integrals and monodromy) such that $\left(^{*}\right)\left(\partial d \mathcal{S} / \partial T_{n}\right) \simeq d \Omega_{n}$, which would make $d \mathcal{S}$ a kind of generating function for all solutions of $(\bullet)$. Thus write at the quasiclassical or averaged level $a_{j}=\oint_{A_{j}} d \mathcal{S} ; a_{j}^{D}=\oint_{B_{j}} d \mathcal{S}$ and stipulate that $\left(\partial a_{j} / \partial T_{n}\right)=0$ and $\left(\partial a_{j}^{D} / \partial T_{n}\right)=\oint_{B_{j}} d \Omega_{n}=\kappa_{j}^{n}(h)$. Now expand $d \mathcal{S}$ as $(\dagger) d \mathcal{S} \simeq \sum_{-\infty}^{\infty} u_{m}(T) d \hat{\Omega}_{m}(h)$ with coefficients $u_{m}(T)$ independent of $h$. Putting $(\dagger)$ into $(*)$ we get

$$
\begin{aligned}
& \frac{\partial d \mathcal{S}}{\partial T_{n}} \simeq \sum_{-\infty}^{\infty}\left(\frac{\partial u_{m}}{\partial T_{n}} d \hat{\Omega}_{m}+u_{m} \frac{\partial d \hat{\Omega}_{m}}{\partial h_{k}} \frac{\partial h_{k}}{\partial T_{n}}\right) \simeq \\
\simeq & \sum_{-\infty}^{\infty}\left(\frac{\partial u_{m}}{\partial T_{n}} d \hat{\Omega}_{m}+u_{m} \sum_{k} \frac{\partial h_{k}}{\partial T_{n}} \sum_{1}^{g} \sigma_{k i}^{m}(h) d \omega_{i}\right)
\end{aligned}
$$

We will write now $\partial_{n} \equiv \partial / \partial T_{n}$ and then upon comparing $\partial_{n} d \mathcal{S} \simeq d \Omega_{n} \simeq d \hat{\Omega}_{n}-\sum_{1}^{g} c_{i}^{n} d \omega_{i}$ with (3.1) one gets (\$o;) $\partial_{n} u_{m}=\delta_{m n} \Rightarrow u_{m}(T)=T_{m}$ along with the formula $\sum_{k} \partial_{n} h_{k}\left(\sum_{-\infty}^{\infty} T_{m} \sigma_{k i}^{m}\right) \equiv$ $\sum_{k} \partial_{n} h_{k} \sigma_{k i}=-c_{i}^{n}$. The last equation represents a version of the Whitham dynamics for $h_{k}\left(\sigma_{k i}\right.$ is defined as indicated). In particular the moduli $h_{k}$ are necessarily $T$ dependent. We note that normally one thinks of Whitham equations as equations for $\partial_{n} \Omega_{k}$ as in (2.4) or as equations for branch points as indicated after (2.6) (here $\partial_{n} \sim \partial / \partial T_{n}$ for slow variables $T_{n}$ ). The branch points are moduli in these hyperelliptic situations so in a general sense one can think of any nonlinear first order PDE in the moduli (e.g. $\left.h_{k}\right)$ as Whitham equations. In the present situation one has in fact

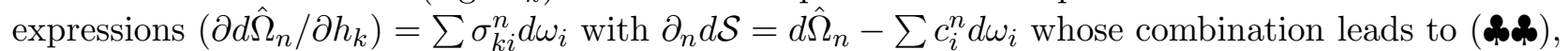
i.e. to $\sum \partial_{n} h_{k} \sigma_{k i}=-c_{i}^{n}$. At this point no restrictions on the number of moduli have been made (but 
see below). We refer also to [2] for another viewpoint on Whitham theory.

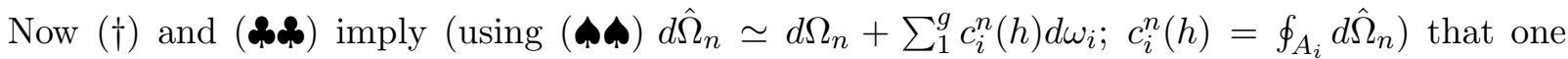
has $d \mathcal{S} \simeq \sum_{-\infty}^{\infty} T_{m} d \hat{\Omega}_{m}(h \mid T) \simeq \sum_{-\infty}^{\infty}\left(T_{m} d \Omega_{m}+T_{m} \sum_{1}^{g} c_{i}^{m} d \omega_{i}\right)$ Integrating this along $A_{j}$ cycles and using (\$\$) again we get $a_{j}=\oint_{A_{j}} d \mathcal{S}=\sum_{-\infty}^{\infty} T_{m} c_{j}^{m}=\sum_{-\infty}^{\infty} T_{m} \oint_{A_{j}} d \hat{\Omega}_{m}$ leading to the desired expression $d \mathcal{S} \simeq \sum_{1}^{g} a_{i} d \omega_{i}+\sum_{-\infty}^{\infty} T_{m} d \Omega_{m}$. Note that $a_{i}=a_{i}(h(T))$ means that the $a_{i}$ seem to be $T$ dependent but the $T$ dependence via $h_{k}$ actually cancels out since $\partial a_{j} / \partial T_{n}=0$ has been stipulated. This means that one can add the $a_{i}$ as additional independent variables to the set of slow times $T_{n}$ and write $d \mathcal{S}=d \mathcal{S}\left(a_{i}, T_{n}\right)$. Upon doing this we should add an equation for $\partial d \mathcal{S} / \partial a_{i}$ to $\left(^{*}\right)$. This can not be postulated arbitrarily since $d \mathcal{S}$ is already defined and hence such equations must be derived. To do this we assume now explicitly $K=g$ so that $\left(\sigma_{k i}\right)$ in (\$\$) will be a square matrix. Then some calculation yields $\left(\partial h_{k} /\left.\partial a_{j}\right|_{T=c}\right)=\left(\sigma_{j k}\right)^{-1}(h)$ from which one can conclude that $d \mathcal{S}=\sum_{-\infty}^{\infty} T_{m} d \hat{\Omega}_{m}=\sum_{1}^{g} a_{i} d \omega_{i}+\sum_{-\infty}^{\infty} T_{n} d \Omega_{n}$ with $\partial d \mathcal{S} / \partial a_{i}=d \omega_{i}$ and $\partial_{n} d \mathcal{S}=d \Omega_{n}$. We can also write now $h_{k}\left(a_{i}, T_{n}\right)$ once the $a_{i}$ have been established as independent variables. Next one looks at the B-periods of $d \mathcal{S}$ in the form

$$
a_{j}^{D}=\oint_{B_{j}} d \mathcal{S}=\oint_{B_{j}}\left(\sum_{1}^{g} a_{i} d \omega_{i}+\sum_{-\infty}^{\infty} T_{n} d \Omega_{n}\right)=\sum_{1}^{g} a_{i} b_{i j}(h)+\sum_{-\infty}^{\infty} T_{n} \kappa_{j}^{n}(h)
$$

Then evaluating $\partial a_{j}^{D} / \partial a_{i}=\oint_{B_{j}}\left(\partial d \mathcal{S} / \partial a_{i}\right)=\oint_{B_{j}} d \omega_{i}=b_{i j}(h)$ we see from the symmetry $b_{i j}=b_{j i}$ that there is some function $\mathcal{F}$ such that $a_{j}^{D}=\left(\partial \mathcal{F} / \partial a_{j}\right)$. This $\mathcal{F}\left(a_{i}, T_{n}\right)$ is called a logarithm of a quasiclassical tau function or a prepotential. The arguments are essentially the moduli of solutions to the original Toda like or low energy YM dynamical system with $a_{i}\left(h_{k}\right)$ parametrizing the moduli of the curve $\Sigma_{g}$ and $T_{n}$ moduli for coordinate systems in the vicinity of punctures. It turns out that the point $T_{n}=0$ for all $n$ is usually singular (note $\sigma_{k i}=\sum_{-\infty}^{\infty} T_{m} \sigma_{k i}^{m}$ vanishes when all $T_{n}=0$ so $\sigma_{i k}^{-1}$ does not exist). One can give explicit formulas for many quantities in terms of contour integrals which we omit here (cf. [23]) but we do note that $\mathcal{F}$ is a homogeneous function of degree two, namely $\mathcal{F}=\frac{1}{2}\left(\sum_{1}^{g} a_{i}\left(\partial \mathcal{F} / \partial a_{i}\right)+\sum_{-\infty}^{\infty} T_{n} \partial_{n} \mathcal{F}\right)$.

\section{SYMPLECTIC GEOMETRY}

The study of TFT and LG theories in connection with integrable systems and Whitham dynamics has an extensive literature (we mention here only [1, 0, 9, 11, 12, 15, 21, 27, 31]). There are many visible connections between $F$ in the quasiclassical tau function $\tau=\exp (F)$ for $\mathrm{KP} /$ Toda theories and the prepotential $\mathcal{F}$. Further, $\mathcal{F}$ and the LG (super)potential $W$ for example are also related to the Hodge-Kähler special geometry in the $N=2$ wonderland (cf. [9, 19, 21]). Recently in [6] it was shown that the theory of the prepotential for $N=2$ susy YM leads to WDVV (WittenDijkgraaf- Verlinde-Verlinde) equations as in TFT (cf. [15]). Also in [17] one uses duality theory as in SW theory to produce a Legendre transform relating $x, \mathcal{F}$, and the probability density $|\psi|^{2}$ for the Schrödinger equation; this is suggested as a way to quantize geometry. Thus there are many directions and connections.

In this section we discuss briefly some constructions from [26] which were in part spelled out more completely in [9]. The paper [26] gives a particularly appropriate development of the theory of integrable systems in connection with $N=2$ susy YM. The role of $d \lambda_{S W}$ as an action-period creature is emphasized and for hyperelliptic Toda systems of the type arising in $N=2$ susy YM the theory seems quite complete. For systems of Calogero-Moser type with underlying elliptic curve there is much less information. In any event one outstanding feature of this work is to show how 
much can be based on the BA functions $\psi$ and $\psi^{*}$. One begins with a universal configuration space which is a moduli space of Riemann surfaces with $N$ punctures $P_{\alpha}$ (better thought of as marked points) and two Abelian integrals $E$ and $Q$ with poles of order at most $n=\left(n_{\alpha}\right)$ and $m=\left(m_{\alpha}\right)$ at the $P_{\alpha}$. One defines an $n_{\alpha}$-jet $\left[z_{\alpha}\right]_{n_{\alpha}}$ of coordinates near a puncture $P_{\alpha}$ to be an equivalence class of coordinates $z_{\alpha}$ with $z_{\alpha} \equiv z_{\alpha}^{\prime}$ if $z_{\alpha}^{\prime}=z_{\alpha}+O\left(z_{\alpha}^{n_{\alpha}+1}\right)$; this space of jets has dimension equal to $n_{\alpha}$. For $[z]_{n}$ a jet near $P_{1}$ we define an Abelian integral $Q$ as a pair $\left(d Q, c_{Q}\right)$ where $d Q$ is a meromorphic differential on the surface $\Sigma$ and $Q=\sum_{-m}^{\infty} c_{k} z^{k}+c_{Q}+R^{Q} \log (z)$ if $d Q=d\left(\sum_{-m}^{\infty} c_{k} z^{k}\right)+R^{Q}(d z / z)$. By integrating $d Q$ along paths one extends the Abelian integral $Q$ holomorphically to a neighborhood of any point in $\Sigma /\left\{P_{1}, \cdots, P_{N}\right\}$. The analytic continuation will depend in general on the path. Let us concentrate on $N=1$, with $(n, m)=(n, 1)$ fixing the singularities for $E$ and $Q$, and take $d E \sim d\left(z^{-n}+O(z)\right) d z \sim d \Omega_{n}$ with $d Q \sim d\left(z^{-1}+O(z)\right) \sim d p$ (necessarily $R_{1}^{E}=R_{1}^{Q}=0$ ). For comparison purposes below we will take $n=3$ later as well. The universal configuration space is defined as $\mathcal{M}_{g}(n, 1)=\left\{\Sigma, P_{1},[z]_{n}, E, Q\right\}$ where $\Sigma$ is a smooth Riemann surface of genus $g$; there are then $3 g-3$ parameters for the Riemann surface, $n$ for the jet $[z]$ or order $n$, and $g+1$ each for $E$ and $Q$, giving $5 g+n$ parameters (note e.g. $E$ has one parameter for $c_{E}$ and $g$ for the holomorphic differentials which can be added without modifying the singular expansion. For local coordinates one chooses now $5 g+n$ objects $\left(P \sim P_{1}, z \sim z_{1}\right)$

$$
\begin{gathered}
T_{k}=\frac{1}{k} \operatorname{Res}_{P}\left(z^{k} Q d E\right),(k=1, \cdots, n) ; \tau_{A_{i}, E}=\oint_{A_{i}} d E ; \\
\tau_{B_{i}, E}=\oint_{B_{i}} d E ; \tau_{A_{i}, Q}=\oint_{A_{i}} d Q ; \tau_{B_{i}, Q}=\oint_{B_{i}} d Q ; a_{i}=\oint_{A_{i}} Q d E
\end{gathered}
$$

where $i=1, \cdots, g$ and it is $Q d E=p d E$ which will play the role of $d \lambda_{S W}$ here.

Now let $\mathcal{D}$ be the open set in $\mathcal{M}_{g}(n, 1)$ where the zero divisors of $d E$ and $d Q$ do not intersect (i.e. the sets $\{\gamma ; d E(\gamma)=0\}$ and $\{\gamma ; d Q(\gamma)=0\}$ do not intersect). It is proved that near each point in $\mathcal{D}$ the $5 g+n$ functions $T_{k}, \tau_{A_{i}, E}, \tau_{B_{i}, E}, \tau_{A_{i}, Q}, \tau_{B_{i}, Q}, a_{i}$ have linearly independent differentials, and thus define a local holomorphic coordinate system. Further the joint level sets of these functions (omitting the $a_{i}$ ) define a smooth g-dimensional foliation of $\mathcal{D}$, independent of the choices made in defining the coordinates themselves. Now $\mathcal{M}_{g}=\mathcal{M}_{g}(n, 1)$ can be taken as a base space for two fibrations $\mathcal{N}^{g}$ and $\mathcal{N} ; \mathcal{N}^{g}$ has fiber $S^{g}(\Sigma) \simeq J(\Sigma)=$ Jacobian variety (via the Abel map $\left.\left(\gamma_{1}, \cdots, \gamma_{g}\right) \rightarrow \sum_{i=1}^{g} \int_{P}^{\gamma_{i}} d \omega_{j}\right)$ and $\mathcal{N}$ has fiber $\Sigma$ (all over a point $\left.\left(\Sigma, P,[z]_{n}, E, Q\right) \in \mathcal{M}_{g}\right)$. We consider only leaves $\mathcal{M}$ of the foliation of $\mathcal{D}$ indicated above and look at the fibration $\mathcal{N}$ or $\mathcal{N}^{g}$ over the base $\mathcal{M}$. One wants to define a symplectic form $\omega_{\mathcal{M}}$ on $\mathcal{N}^{g}$. First, although $E$ and $Q$ are multivalued on the universal fibration their differentials are well defined on $\mathcal{N}$. The idea here is that $E$ and $Q$ are well defined near $P_{1}$ and their analytic continuations by different paths can only change by multiples of their residues or periods along closed cycles. But on a leaf of the foliation the ambiguities remain constant and disappear upon differentiation. Hence one has differentials $\delta E$ and $\delta Q$ on the fibrations which reduce to $d E$ and $d Q$ acting on vectors tangent to the fiber. One can trivialize the fibration $\mathcal{N}$ with the variables $a_{1}, \cdots, a_{g}$ along the leaf $\mathcal{M}$ and e.g. $E$ along the fiber. Then $d Q$ coincides with $(d Q / d E) d E$ where $d E$ is viewed as one of the elements of the basis of one forms for $\mathcal{N}$ and the full differential is $\delta Q=d Q+\sum_{1}^{g}\left(\partial Q / \partial a_{i}\right) d a_{i} \equiv d Q+\delta^{E} Q$ (also one takes $c_{E}=0$ ).

Now if one considers the full differential $\delta(Q d E)$ on $\mathcal{N}$ it is readily seen that it is well defined despite the multivaluedness of $Q$. In fact the partial derivatives $\partial_{a_{i}}(Q d E)$ along the base $\mathcal{M}$ are holomorphic since the singular parts of the differentials as well as the ambiguities are all fixed. In particular $\left(\partial / \partial a_{i}\right)(Q d E)=d \omega_{i}$ where $d \omega_{i}$ is a basis of normalized holomorphic differentials $\oint_{A_{i}} d \omega_{j}=\delta_{i j}$ with $\oint_{B_{i}} d \omega_{j}=b_{i j}$. To see this note that it is implicit in 4.1) since by definition of 
the $a_{i},\left(\partial a_{i} / \partial a_{j}\right)=\delta_{i j}=\oint_{A_{i}}\left(\partial(Q d E) / \partial a_{j}\right)$ which implies $\left(\partial(Q d E) / \partial a_{j}\right)=d \omega_{j}$. Formally then one defines $\omega_{\mathcal{M}}$ on $\mathcal{N}^{g}$ via

$$
\omega_{\mathcal{M}}=\delta\left(\sum_{1}^{g} Q\left(\gamma_{i}\right) d E\left(\gamma_{i}\right)\right)=\sum_{1}^{g} \delta Q\left(\gamma_{i}\right) \wedge d E\left(\gamma_{i}\right)=\sum_{1}^{g} d a_{i} \wedge d \omega_{i}
$$

The first expression seems formally reasonable on $\mathcal{N}^{g}$ and the last appears to be a calculation of the form $\omega_{\mathcal{M}}=\delta(Q d E)=\sum_{1}^{g} d a_{i} \wedge\left[\partial(Q d E) / \partial a_{i}\right]=\sum_{1}^{g} d a_{i} \wedge d \omega_{i}$. Now go to KP for illustration and background. One can work with a (nonspecial) divisor $\left(\gamma_{1}, \cdots, \gamma_{g}\right)$ giving rise to quasiperiodic functions of $t=\left(t_{n}\right) 1 \leq n<\infty$, of the form $u_{i, n} 1 \leq i \leq n-2,2 \leq n<\infty$, which arise as solutions of an integrable hierarchy. The BA function is defined as a meromorphic function away from $P$ with simple poles at the $\gamma_{i}(1 \leq i \leq g)$ and an essential singularity at $P$ of the form $\psi(t, z)=\exp \left(\sum_{1}^{\infty} t_{n} z^{-n}\right)\left(1+\sum_{1}^{\infty} \xi_{i}(t) z^{i}\right)$. There is a Lax operator (in general one for each puncture) $L_{n}=\partial^{n}+\sum_{0}^{n-2} u_{i, n} \partial^{i}$ with $\left(\partial / \partial t_{n}-L_{n}\right) \psi(t, z)=0\left(\partial=\partial_{x}, x=t_{1}\right)$. Thus there is a map $\left\{\Sigma, P, z, \gamma_{1}, \cdots, \gamma_{g}\right\} \rightarrow\left\{u_{i, n}(t)\right\}$. An explicit form for the BA function for KP is given in [26] but it involves normalizations $\Re \oint_{C} d \Omega_{n}=0$ for any cycle $C$ and is more complicated in appearance that our previous expression in Section 2 for example. Similarly the dual BA function $\psi^{*}$ is defined as before (and denoted by $\psi^{\dagger}$ in [26]). We note that for the Toda lattice one takes $N=2$ punctures.

An element in $\mathcal{N}^{g}(n, 1)$ gives rise to a datum in a space $\hat{\mathcal{N}}^{g}$ via $\Xi:\left(\Sigma, P,[z]_{n}, E, Q, \gamma_{1}, \cdots, \gamma_{g}\right) \rightarrow$ $\left(\Sigma, P, z, \gamma_{1}, \cdots, \gamma_{g}\right) \rightarrow\left\{\left.u_{i, n}(t)\right|_{i=1} ^{n-2}\right\} \in \hat{\mathcal{N}}^{g}$. Take a real leaf $\mathcal{M}$ (i.e. $\Re \oint_{C} d E=\Re \oint_{C} d Q=0$ for all cycles $C$ on $\Gamma$ ) and write still $d Q \sim d \Omega_{1}=d p$ and $d E \sim d \Omega_{n}$ (with real normalizations). We also take $t_{1} \sim x$. One wants now to express $\omega_{\mathcal{M}}$ in terms of forms on the space of functions $\left\{u_{i, n}(t)\right\}$. First the $u_{i, n}(t)$ can be written in terms of the asymptotic BA coefficients $\xi_{i}$ and one knows that $\left(\partial_{x} \psi / \psi\right)=z^{-1}+\sum_{1}^{\infty} h_{s} z^{s}$ and $\left(\partial_{x} \psi^{*} / \psi^{*}\right)=-z^{-1}+\sum_{1}^{\infty} h_{s}^{*} z^{s}$ (cf. [10] and arguments before (2.6) where e.g. $\partial=L+\sum_{1}^{\infty} \sigma_{j}^{1} L^{-j}$ which implies e.g. $(\partial \psi / \psi)=\lambda+\sum_{1}^{\infty} \sigma_{j}^{1} \lambda^{-j}$ for $\left.\lambda \sim z^{-1}\right)$. In any event the first $n-1$ coefficients $h_{s}, h_{s}^{*}$, or $\sigma_{j}^{1}$ are differential polynomials in the $u_{i, n}$ (initial data $\left.\xi_{s}(t)\right|_{x=0}=\phi_{s}\left(t_{2}, \cdots\right)$ determine $\xi_{s}$ for $\left.s \leq n-1\right)$. Writing $H_{s}=<h_{s}>$ one gets now $p=z^{-1}+\sum_{1}^{\infty} H_{s} z^{s}$ (cf. [7]). We note that one uses $<>_{x}$ and $<>_{x y}$ averaging at various places in [26] but generically this should correspond to ergodic averaging.

A result in [26] now asserts that for $\mathcal{N}^{g}$ the Jacobian bundle over a real leaf $\mathcal{M}$ of the moduli space $\mathcal{M}_{g}(n, 1)$ the symplectic form $\omega_{\mathcal{M}}$ can be written as

$$
\omega_{\mathcal{M}}=-\operatorname{Res}_{P} \frac{<\delta \psi^{*} \wedge \delta L \psi>}{<\psi^{*} \psi>} d p=n \sum_{1}^{n-2}<\delta h_{s} \wedge \int^{x} \delta^{*} h_{n-s}^{*}>
$$

where the $h_{s}, h_{s}^{*}$ are differential polynomials as above, and the differential forms $\delta h_{s}$ and $\delta^{*} h_{s}^{*}$ are defined via $\delta h_{s}=\sum_{i=0}^{n-2} \delta u_{i, n} \sum_{\ell}(-\partial)^{\ell}\left(\partial h_{s} / \partial u_{i, n}^{(\ell)}\right)$ and $\delta^{*} h_{s}^{*}=\sum_{i=0}^{n-2} \delta u_{i, n}^{(\ell)} \sum\left(\partial h_{s}^{*} / \partial u_{i, n}^{(\ell)}\right)$.

This is a fascinating result but the proof in [26] requires some embellishment. First one must come to terms with an expression (\$\$) $\delta E=\delta p(d E / d p)+\left(\left\langle\psi^{*} \delta L_{n} \psi\right\rangle /\left\langle\psi^{*} \psi\right\rangle\right)$ where

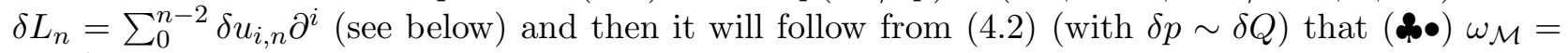
$-\sum_{1}^{g}\left(\left\langle\psi^{*} \delta L_{n} \psi>/<\psi^{*} \psi>\right)\left(\gamma_{s}\right) \wedge \delta p\left(\gamma_{s}\right)\right.$. The formula (\$\$N) is asserted to come from 25] but to see this one has to interpret $\delta u_{i, n}$ as arising from $\epsilon \partial_{X} U_{i, n}(X, T)$ in the first order term. Indeed we can look at (2.5) with the last two terms absent on the leaves of our foliation and written generically for $L_{n} \sim \Omega_{n}$ as $(\bullet \mathbf{\bullet})<\psi^{*}\left(L_{n}^{1} \partial_{X} L_{k}-L_{k}^{1} \partial_{X} L_{n}\right) \psi>=\partial_{X} \Omega_{k}<\psi^{*} L_{n}^{1} \psi>-\partial_{X} \Omega_{n}<\psi^{*} L_{k}^{1} \psi>$. Then for $L_{n}=L_{3} \sim \Omega_{3}$ and $L_{k}=L_{1} \sim p$ one has $L_{1}=\partial, \delta L_{1} \sim \partial_{X} L_{1}=0, L_{1}^{1}=1$ and $-<\psi^{*} \partial_{X} L_{3} \psi>=$ $\partial_{X} p<\psi^{*} L_{3}^{1} \psi>-\partial_{X} \Omega_{3}<\psi^{*} \psi>$. Written in terms of $\delta p \sim \epsilon \partial_{X} p, \delta L_{3} \sim \epsilon \partial_{X} L_{3}$, etc. one obtains $\delta \Omega_{3}=\delta p\left(<\psi^{*} L_{3}^{1} \psi>/<\psi^{*} \psi>\right)+\left(<\psi^{*} \delta L_{3} \psi>/<\psi^{*} \psi>\right)$ while from (2.6) (with $\left.L_{3}^{1} \sim A^{1}\right)$ we 
have $\left(<\psi^{*} L_{3}^{1} \psi>/<\psi^{*} \psi>\right)=\left(d \Omega_{3} / d p\right)$, or generically $\left(d \Omega_{n} / d p\right)=\left(<\psi^{*} L_{n}^{1} \psi>/<\psi^{*} \psi>\right)$, and (\$\$) follows. The formula $(\bullet \mathbf{A})$ can also be used for a more general theorem in [26]. Thus (\$•) holds and writing $\gamma_{s}(t)$ for the zeros of $\psi$ away from $P$ (corresponding to the fixed poles $\gamma_{s}$ for $t=0$ ) one can pick $t_{1}, \cdots, t_{g}$ (generically) as times for which the flows $\gamma_{s}(t)$ are independent and use them as coordinates on $S^{g}(\Sigma)$. These can be transferred to the system of coordinates $\left.f\left(\gamma_{1}\right), \cdots, f\left(\gamma_{g}\right)\right)$ for $f$ an Abelian integral on $\Sigma$ via $\left(\partial / \partial t_{i}\right) f(\gamma(t))=\operatorname{Res}_{\gamma(t)}\left[\left(\partial / \partial t_{i}\right) \psi(t, z) / \psi(t, z)\right] d f$. In the present case, writing $\left(\delta_{t} \psi / \psi\right)=\sum_{1}^{g}\left(\partial_{j} \psi / \psi\right) d t_{j}$ and using $\sum \operatorname{Res}_{\gamma_{s}}=-\operatorname{Res}_{P}$ one obtains $\omega_{\mathcal{M}}=\operatorname{Res}_{P}\left[\left(\left.\left\langle\psi^{*} \delta L_{n} \psi>/\left\langle\psi^{*} \psi\right\rangle\right) \wedge\left(\delta_{t} \psi / \psi\right)\right|_{t=0}\right] d p\right.$. It is not unnatural to see here an apparent change in the number of parameters. Now note that $\psi^{*} \delta L_{n} \psi=\psi^{*} \sum_{0}^{n-2} \delta u_{i, n} \partial^{i} \psi=\sum_{0}^{n-2} \delta u_{i, n} \psi^{*} \partial^{i} \psi$ and an easy calculation gives $\left(\partial^{j} \psi / \psi\right)=z^{-j}\left(1+\sum_{1}^{\infty} c_{j p} z^{p}\right)$; this implies $\left(\psi^{*}\left(\delta L_{n} \psi\right) /<\psi^{*} \psi>\right) d p=$ $-\sum_{1}^{\infty} \delta J_{s} z^{-n-1+s} d z$ for coefficients $\delta J_{s} \sim$ linear combinations of the $\delta u_{i, n}$ (recall $\left(d p /<\psi^{*} \psi>\right)=$ $\left.O\left(z^{-2}\right)\right)$. Averaging now gives $\left(<\psi^{*}\left(\delta L_{n} \psi>/<\psi^{*} \psi>\right) d p=-\sum_{1}^{\infty}<\delta J_{s}>z^{-n-1+s} d z\right.$ so (\$\$)) implies $\delta H_{s}=<\delta J_{s}>=0$ for $1 \leq s \leq n-1$. Hence $\omega_{\mathcal{M}}=-\sum_{1}^{g}<\delta J_{j+n}>\wedge d t_{j}$. Further from [10, 11] one has $\left(\partial_{j} \psi^{*} / \psi^{*}\right)=-\lambda^{j}-\sum_{1}^{\infty} \sigma_{s}^{j} \lambda^{-s}=-z^{-j}+\sum_{1}^{\infty} h_{s, j}^{*} z^{s}$ and a calculation gives $\delta J_{j+n}(t)=$ $\sum_{1}^{n-1} \delta J_{s} h_{n-s, j}^{*}$; hence $\omega_{\mathcal{M}}=-\operatorname{Res}_{P}\left(<\delta_{t} \psi^{*} \wedge \delta L_{n} \psi>/<\psi^{*} \psi>\right) d p$ holds and an argument is suggested in [26] to extend $\delta_{t}$ to a full $\delta$ so that the first formula in (4.3) is proved. For the second formula in (4.3) one looks at $\delta \log \psi^{*}=\delta\left(c\left(t_{i}, i \geq 2\right)+\int_{x_{0}}^{x} \partial_{x} \log \psi^{*}\right)=\delta \sum_{1}^{\infty}\left(c_{s}\left(t_{i}, i \geq 2\right)+\int_{x_{0}}^{x} h_{s}^{*} z^{s} d x\right)$. This implies $\left.-\operatorname{Res}_{P}\left(<\left(\delta \log \psi^{*}\right) \wedge\left(\psi^{*} \delta L_{n} \psi\right)>\right) /<\psi^{*} \psi>\right)=\omega_{\mathcal{M}}=-\sum_{1}^{n-1}<\delta J_{s} \wedge \int_{x_{0}}^{x} \delta^{*} h_{n-s}^{*} d x>$. By definitions $\delta J_{s}$ does not contain variations of derivatives of $u_{i}$ so we can write $\delta J_{s}=-n\left(\partial h_{s} / \partial u\right) \delta u$ and this gives the second identity in (4.3). We note here that there is a little interplay between $\delta \sim \epsilon \partial_{X}$ and $X=\epsilon x$ in the last two lines. The examples in 26] have some curious features but in any event one should keep in mind the conditions $\delta H_{s}=0$ when selecting functions. One notes that of course the symplectic structure involving $\delta h_{s}, \delta^{*} h_{s}^{*}$, etc. does not include the time dynamics so $\mathrm{KdV} \sim L_{2}=\partial^{2}+u$ for example and Bousinesq $\sim L_{3}=\partial^{3}+u \partial+v$. Adaptions and applications of the above fibration framework to $S U(n), N=2$ susy YM theory are also given in [26].

\section{References}

[1] S. AOYAMA \& Y. KODAMA, Topological LG theory with a rational potential and the dispersionless KP hierarchy, hep-th 9505122

[2] O. BABELON, D. BERNARD, \& F. SMIRNOV, Null vectors in integrable field theory, hep-th 9606068

[3] E. BElokolos, A. BOBEnKO, V. ENOLSKIJ, A. ITS, \& V. MATVEEV, Algebro-geometric Approach to Nonlinear Integrable Equations, Springer, New York (1994)

[4] A. BILAL, Duality in N=2 susy SU(2) YM theory, hep-th 9601007

[5] A. BLOCH \& Y. KODAMA, Dispersive regularization of the Whitham equations for the Toda lattice, SIAM J. appl. Math., 52, 909-928 (1992)

[6] G. BONELLI \& M. MATONE, Nonperturbative renormalization group equation and beta function in N=2 susy YM, hep-th 9605090

[7] R. CARROLL, Remarks on the Whitham equations, solv-int 9511009, to appear

[8] R. CARROLL, Topics in Soliton Theory, North-Holland, Amsterdam (1991)

[9] R. CARROLL, Assorted lecture notes on KdV, KP, TFT, susy, integrability, Riemann surfaces, matrix models, SW theory, etc., 542 pages, Univ. Illinois (1995-1996)

[10] R. CARROLL, Symmetries, Sato theory, and tau functions, Applicable Anal., 56, 147-164 (1995)

[11] R. CARROLL, Remarks on dispersionless KP, KdV, and 2-D gravity, J. nonlin. Sci., 4, 519-544 (1994) 
[12] R. CARROLL \& Y. KODAMA, Solution of the dispersionless Hirota equations, J. Phys. A, 28, 6373-6387 (1995)

[13] R. DONAGi \& E. WITTEN, Susy YM theory and integrable systems, Nucl. Phys. B, 460, 299-334 (1996)

[14] B. DUBROVIN, Theta functions and nonlinear equations, Russ. Math. Surveys, 36, 11-92 (1981)

[15] B. DUBROVIN, Geometry of 2-D field theories, in Integrable Systems and Quantum Groups, pp. 120-348, Springer, New York (1996)

[16] T. EGUCHI \& S. YANG, Prepotential of N=2 susy gauge theories and soliton equations, Mod. Phys. Lett. A, 11, 131-138 (1996)

[17] A. FARAGGI \& M. MATONE, A statistical interpretation of space and classical-quantum duality, hep-th 9606063

[18] H. FLASCHKA, M. FOREST, \& D. MCLAUGHLIN, Multiphase averaging and the inverse spectral solution of the KdV equation, Comm. Pure Appl. Math., 33, 739-784 (1980)

[19] P. FRÉ \& P. SORIANI, The $N=2$ Wonderland, World Scientific, Singapore (1995)

[20] F. FUCITO, A. GAMBA, M. MARTELLINI, \& O. RAGNISCO, Nonlinear WKB analysis of the string equation, Inter. J. mod. Phys. B, 6, 2123-2147 (1992)

[21] C. GÓMEZ \& E. LÓPEZ, Topology and strings: Topics in N=2, hep-th 9411056

[22] A. GORSKY, I. KRICHEVER, A. MARSHAKOV, A. MIRONOV, \& A. MOROZOV, Integrability and SW exact solutions, Phys. Lett. B, 355, 466-474 (1995)

[23] H. ITOYAMA \& A. MOROZOV, Integrability, prepotential, and SW theory, hep-th 9511126; 9512161; 9601168

[24] A. KLEMM, W. LERCHE, \& S. THEISEN, Nonperturbative effective action of N=2 susy gauge theories, hep-th 9505150

[25] I. KRICHEVER, Method of averaging for 2-D integrable equations, Funkts. Anal. Prilozh., 22, 200-213 (1988)

[26] I. KRICHEVER \& D. PHONG, On the integrable geometry of soliton equations and N=2 susy gauge theories, hep-th 9604199

[27] I. KRICHEVER, Algebraic-geometric methods in the theory of integrable equations and their perturbations, Acta applic. math., 39, 93-125 (1995)

[28] P. LAX, C. LEVERMORE, \& S. VENAKIDES, The generation and propagation of oscillations in dispersive initial value problems and their limiting behavior, in Important Developments in Soliton Theory, pp. 205-241, Springer New York (1993)

[29] T. NAKATSU \& K. TAKASAKI, Whitham-Toda hierarchy and N=2 susy YM theory, Mod. Phys. Lett. A, 11, $157-168(1996)$

[30] N. SEIBERG \& E. WITTEN, EM duality, monopole condensation, and confinement in N=2 susy YM theory, Nucl. Phys. B, 426, 19-52 (1994)

[31] K. TAKASAKI \& T. TAKEBE, Integrable hierarchies and dispersionless limit, Rev. math. Phys., 7, 743-808 (1995)

[32] K. TAKASAKI \& T. NAKATSU, Isomonodromy deformations and susy gauge theories, hep-th 9603069 